\title{
Production de l'espace urbain dans les pays du sud
}

\section{Urbanization in Southern Countries}

Philippe Plas - Docteur en sociologie, Maitre de Conférences en Sociologie, Université Paris 13 Sorbonne Paris Cité, Laboratoire CERAL, Directeur du Master de Conseil aux Collectivités Territoriales en Stratégie de Développement Durable. E-mail: philippeplas9@hotmail.fr

\section{Résumé}

Les pays émergents du Sud connaissent actuellement une phase importante de leur processus d'urbanisation. Les quatre logiques qui président à la production de l'espace urbain : logique économique, logique politique, logique sociale et logique culturelle s'y articulent dans des configurations singulières. Une des caractéristiques essentielles de cette configuration c'est la relative faiblesse de la logique politique qui de ce fait subit la domination de la logique économique. Ces sociétés très inégalitaires produisent des villes tripartites ou à côté des espaces soumis à la logique économique subsistent des espaces dans lesquels les logiques traditionnelles contribuent puissamment à la production de ces espaces. L'enjeu de ces transformations est bien de savoir dans quelle mesure la logique politique appuyée sur la logique sociale parviendra à s'imposer aux forces dominantes de la logique économique.

\section{Mots-clé}

Urbanisation. Production de l'espace urbain. Configuration. Logique politique.

\begin{abstract}
South emergent countries are curently in a important phase of their urbanization process. The four logics that lead the production of the urban space : The economic logic, the politic logic, the social logic and the cultural logic are articulates in weird configurations over there. One of the essential feature of this configuration is the relative weakness of the political logic, which thereby undergoes the domination of the economic logic. These very unegalitarian societies product tripartite cities where, near the spaces that are led by the economic logic, remain spaces where the traditional logic strongly contributes to the production of these spaces. The challenge of these transformations is to know in wich measures the political logic, resting on the social logic will achieve to impose itself to the dominant forces of the economic logic.
\end{abstract}

\section{Keywords}

Urbanisation. Production of the urban space. Configuration. Political logic 
L'urbanisation accélérée est une des caractéristiques les plus incontestables du monde contemporain. Depuis le début du $19^{\text {ème }}$ siècle la démographie urbaine n'a fait que croître. Il y a incontestablement une corrélation entre le processus d'industrialisation et le processus d'urbanisation. La croissance urbaine s'est en effet manifestée, dans un premier temps, dans les premiers pays concernés par la révolution industrielle, l'Angleterre et la France, rapidement suivis par les autres puissances industrielles au fur et à mesure de leur développement, Allemagne et Etats-Unis en premier lieu.

Certains pays du Sud ont depuis longtemps également été touchés par des phénomènes d'urbanisation. Il s'agissait cependant d'une urbanisation d'une nature très différente de celle observée au Nord. Elle était principalement la conséquence d'une démographie exponentielle qui affectait ces pays dont la population croissait à un rythme considérable. Le géographe brésilien Milton Santos [Santos 1971] parlait à ce propos de « l>urbanisation démographique des Pays En Développement » qu'il opposait à « l'urbanisation technologique» des pays industrialisés. Les sociétés traditionnelles rurales se trouvaient dans l'impossibilité d'absorber l'accroissement de leur population, celle-ci se trouvait alors contrainte de quitter la campagne pour gagner la ville. De cette manière se développaient des centres urbains parfois considérables comme Bombay, Le Caire, Mexico ou même Rio de Janeiro. D’ailleurs depuis le milieu du vingtième siècle la rapidité du processus d'urbanisation en cours dans les pays du sud est sans commune mesure avec celle de l'Europe au 19ème siècle: en seulement un quart de siècle, les taux d'urbanisation ont doublé dans nombre de pays d'Afrique subsaharienne ou en Chine tant et si bien qu'aujourd'hui 7 urbains sur dix vivent dans les pays du Sud alors qu'en 19506 sur 10 vivaient dans les pays du Nord.

Le phénomène de croissance urbaine qu'on observe aujourd'hui dans de nombreux pays du Sud est cependant de nature différente. C'est un modèle d'urbanisation inédit qui est à l'œuvre : par les masses de population concernées ; par le degré de concentration des populations citadines dans des métropoles de taille inégalée dans l'histoire; par le contexte économique des villes des pays du Sud et par le rôle accru des populations dans la production de l'urbain.

On peut en effet considérer que dans le contexte actuel de mondialisation ce qu'Henri Levèbvre appelait la « révolution urbaine » [Lebvèvre 1970] gagne l'ensemble de la planète [Veron 2006] et tout particulièrement les pays émergents comme la Chine [Boquet 2009] ou le Brésil (87\% du total de la population en 2010 y est urbaine). Ces pays qui se trouvent dans une situation de transition voient émerger un modèle d'urbanisation, de production de l'espace, qui correspond à cette situation particulière. Pour une part il conserve certains aspects de 
l'urbanisation liée aux migrations des campagnes sous la pression démographique mais par ailleurs il faut y voir la conséquence de l'inscription de ces pays dans les logiques de la révolution industrielle dans un contexte de mondialisation. Les villes du Sud dans les pays émergents entrent progressivement dans une dynamique urbaine tout à fait nouvelle [Allemand 2012]. La ville en effet est toujours un extraordinaire révélateur des transformations qui affectent une société. Ces transformations produisent toujours des effets sur les modalités d'organisation de l'espace urbain. Les mutations socioéconomiques s'inscrivent dans l'espace en provoquant des réorganisations des espaces territoriaux et tout particulièrement de l'espace urbain. Cependant il ne s'agit pas d'un simple mécanisme de reflet. La ville n'est pas le réceptacle passif des rapports socioéconomiques dominants. Les rapports sociaux urbains disposent d'une véritable autonomie. Les changements socioéconomiques mettent la ville en mouvement mais c'est dans ce mouvement que s'inventent des rapports sociaux qui ensuite font retour dans l'ensemble de la société. La ville devient la matrice des transformations culturelles qui vont alors affectées la société et la société urbaine comme le disait aussi H.Lebvèvre se limite de moins en moins à la ville. Pour lui les sociétés occidentales sont de plus en plus des sociétés urbaines dans leur totalité à la condition de comprendre la notion de société urbaine comme un modèle culturel et pas simplement la culture de ceux qui vivent en ville. Dans le contexte actuel de la mondialisation on peut considérer que ce phénomène tend actuellement à s'étendre à l'ensemble du monde notamment émergent.

Ce processus d'urbanisation présente des spécificités liées aux différentes réalités culturelles caractérisant les différents lieux où il se produit ; cependant il est possible de mettre en évidence des régularités fortes dans les modalités selon lesquelles s'organisent la production et le changement de la ville, et ce plus particulièrement dans les pays occidentaux puis dans les pays du Sud émergents. Comme l'a bien souligné Thierry Paquot : « la ville de la modernité née en Europe au $19^{\text {ème }}$ siècle, marque une rupture historique » et surtout : « La révolution urbaine annoncée par Henri Lebvèvre en 1970 s'accomplit sous nos yeux dans les pays du tiers monde alors qu'elle s'achève dans la plupart des pays industriels et postindustriels » [Paquot 2000 p.6]. Cette dynamique si, répétonsle, elle prend bien sur des formes différentes selon les pays, présente cependant une logique interne relativement universelle. C'est cette logique qui servira de fondement théorique à notre démarche.

Nous aborderons cette question en deux parties. Tout d'abord il me semble indispensable de préciser le cadre conceptuel de l'analyse. En effet la production de la ville constitue un champ de réflexion extrêmement complexe trop souvent 
abordé dans une démarche impressionniste ou de manière très idéologique, pourtant il est indispensable d'affermir la pensée théorique sur la ville, de lui donner une véritable ossature conceptuelle; c'est ce que nous essaierons de faire dans la première partie de cet article. Après ce rappel dans la seconde partie nous essaierons de voir ce que ce schéma théorique révèle de la production urbaine dans les villes du sud et plus particulièrement dans les pays émergents de la zone.

Partout dans le monde, comme dans l'histoire d'ailleurs la ville est concernée par un paradoxe fondamental. Celui-ci oppose d'une part la volonté politique de maîtrise de l'espace urbain et d'autre part l'irrésistible dynamisme urbain qui déjoue toutes les planifications.

\section{Le paradoxe urbain}

Toute réflexion sur la ville se doit en effet de partir d'un constat relativement trivial : à travers l'histoire du phénomène urbain se manifeste une tenace volonté d'organiser et de contraindre l'espace urbain. C'est une constante depuis que la ville existe soit dès ce que Gordon Childe [Gordon Childe 1925] a qualifié de « révolution néolithique » et déjà de révolution urbaine. Trois moments semblent particulièrement symptomatiques de cette logique.

Du point de vu conceptuel on s'accorde à penser que le premier théoricien de la conception de l'espace urbain est le philosophe grecque Hippodamos de Millet qui fut le premier à préconiser le plan géométrique tel qu'il se rencontre aujourd'hui dans les villes américaines par exemple. Mais le premier exemple particulièrement significatif en occident c'est incontestablement la ville romaine [Duby 1980]. La puissance politique de l'empire romain s'est inscrite dans l'espace à travers l'imposition dans tout l'espace méditerranéen d'un modèle urbain unique. Cette ville quadrillée à partir du cardo et du decumanus où au croisement de ces deux axes se tenait le cœur de la cité : temple, édifice politique, forum, salle de justice auxquels s'ajoutaient thermes et amphithéâtres proclamait dans la pierre et dans l'espace la puissance et la magnificence de Rome. Et pourtant jamais et nulle part Rome ne sut et ne put contrôler l'effervescence urbaine. De toute part la ville vivante va proliférer, s'étendre, échappant à tout contrôle, même la limitation de la hauteur des immeubles ne pourra pas être respectée y compris au cœur de la ville centre ou les « insulae » prolifèreront sans contrôle. Rome, au fait de sa puissance, maittre du monde ne pourra jamais maitriser la prolifération urbaine.

Le même scénario se reproduira quand en occident s'installera la ville médiévale [Le Goff 1998]. La ville qui renaît autour de l'an mil après plusieurs 
siècles de régression urbaine en occident ne s'inscrit pas dans la continuité de la ville romaine, même si souvent elle se déploie sur les mêmes sites, c'est une ville circulaire qui se caractérise par une certaine spécialisation fonctionnelle de l'espace. Les différentes activités artisanales ou commerçantes organisées en corporation sont regroupées par quartier. La ville forme une sorte de spirale du centre vers la périphérie. C'est aussi une ville close. Elle se tient à l'intérieur de ses murs et ferme ses portes chaque soir. Cette fermeture exige de maitriser le développement de la ville, son expansion maximum est limitée par le rempart. L'expérience montre que cette maitrise sera toujours impossible. Toujours la ville débordera de ses murs, partout se développeront des faubourgs à l'extérieur de la muraille. Souvent la réponse sera la construction de murailles successives pour suivre l'expansion urbaine. Pas plus la ville médiévale que la ville antique ne pourront maitriser leur expansion ni même finalement leur forme.

Ce sera le cas de Paris par exemple qui comptera pas moins de sept enceintes successives. Même avec les tentatives de la ville Classique [Duby, 1985] de contrôler son développement de donner à voir le pouvoir avec la multiplication des places royales, elle ne parviendra pas à empêcher que se développe en son cœur une ville populaire proliférante. Quand dans une première phase de la révolution industrielle Paris connait une croissance rapide (la ville gagne 500000 habitants entre 1800 et 1850) c'est par un incroyable mécanisme de densification d'élévation, d'occupation des cours que se produit une véritable « réorganisation de l'espace » [Gribaudi, 2014] échappant à toute planification. Il faudra toute la violence de la transformation Haussmannienne pour reprendre la volonté de maitrise et restaurer la volonté politique de piloter le développement urbain. Cela n'empêchera pas que dès la fin du $19^{\text {ème }}$ de nouveaux quartiers populaires apparaissent et débordent l'ordonnancement Haussmannien.

Cette volonté profonde de maitriser la production urbaine connaitra une prolongation théorique et conceptuelle au tournant du $19^{\text {ème }}$ et du $20^{\text {ème }}$ siècle. En effet, à la fin du $19^{\text {ème }}$ siècle apparait un secteur nouveau dans le champ de la pensée : l'urbanisme qui, dans le contexte du positivisme dominant d'alors, se fixe pour objectif de proposer à la fois une approche scientifique de la ville et d'en déduire un modèle rationnel de l'aménagement urbain. Il s'agit là d'un moment essentiel dans le développement de la ville en occident. La trajectoire des villes européennes va se trouver profondément conditionnée par ce moment fondateur.

Cette effervescence idéologique sera formalisée dans la Chartes d'Athènes en 1933 qui va fixer pour près d'un demi-siècle le cadre de l'urbanisme dans le monde (Niemeyer par exemple au Brésil) Ces urbanistes qui se qualifient eux 
même de modernes puisqu'ils forment le Congrès internationale d'Architecture Moderne (CIAM) publient un manifeste rédigé par Le Corbusier mais qui en réalité traduit les conceptions dominantes dans le champ d' l'urbanisme d'alors.

Très rapidement rappelons les postulats théoriques de la démarche :

- L'urbanisme est une science. Penser la ville n'est pas une question d'opinion ou de culture c'est une question de vérité scientifique. De ce postulat fondamental plusieurs corolaires peuvent être déduits. Tout d'abord la conception de la ville est une affaire scientifique donc doit être le fait des experts, des urbanismes, c'est eux qui seuls peuvent dire le vrai en matière de conception urbaine. L'opinion publique ne peut porter des jugements pertinents sur la ville et l'urbain incapables de s'inscrire dans l'avenir ils ne peuvent que répéter les choix du passé. De même les politiques inscrit dans l'espace de l'opinion ne sont pas plus légitimes et en conséquence la ville et l'urbain ne sont pas à proprement parlé des questions politiques.

- Comme toute science la pensée urbanistique travaille dans l'universel, la vérité scientifique est la même partout et en conséquence le modèle de l'urbain et de la ville doit être le même partout à Paris à Schangaï ou à Belèm. On retrouve ce que dans le champ du travail Taylor avait résumé par la formule du « one best way » : il y a un « one best way» urbain que les urbanistes seules connaissent.

Ce modèle va servir de matrice à l'urbanisation en Europe et dans le monde dans la deuxième moitié du vingtième siècle. Au Nord comme au Sud le bilan est plutôt accablant. Aujourd'hui, partout la crise urbaine est présente. La critique est générale. En France, pays centralisé, on a décidé dès 1973 de mettre fin à ce type d'urbanisme hégémonique par une décision du ministre de l'aménagement du territoire Olivier Guichard.

Ce qu'il faut surtout retenir c'est que la ville nulle part ne s'est véritablement laissé contraindre. Toujours et partout elle déborde et échappe à l'ordre de ses promoteurs.

La ville échappe à ses décideurs, c'est une réalité sociale dynamique qui articule des mécanismes variées et complexes. Pour reprendre une expression galvaudée de M. Mauss c'est un phénomène social total, le creuset de l'ensemble des dimensions d'une société. Ce sont les différentes dimensions de ce phénomène et les modalités de leur articulation que nous allons essayer de préciser ici.

\section{Configurations et logiques urbaines}

Comme l'a très fortement démontré Henri Lefebvre [Lefebvre 1974 ] la ville est l'inscription dans l'espace des rapports sociaux dominants. La difficulté 
tient au fait que la notion de rapports sociaux est en réalité extrêmement peu précise. Elle se situe en fait à un niveau très élevé de généralité. Comme le montre très bien B.Latour [Latour, 2005] ce type de social ne désigne en fait rien. Ce que nous voulons essayer de faire ici c'est de considérer ces rapports sociaux dans les différentes logiques qui les traversent. Dans une première approche schématique on peut considérer que dans la production de l'espace urbain il y a principalement quatre logiques qui s'articulent :

- Logique économique

- Logique politique

- Logique sociale

- Logique culturelle.

La ville dans sa forme concrète est le résultat de la combinatoire de ces différentes logiques. Par ailleurs le rythme d'évolution de ces différentes logiques est en général différent. La ville n'est en conséquence que très rarement un espace cohérent. Elle est traversée de contradictions très complexes entre ces différentes logiques qui se combinent et se recombinent sans cesse.

Tout changement dans une de ces logiques entraîne la production d'une nouvelle combinatoire. Analyser et comprendre le phénomène urbain c'est chercher à saisir les formes particulières de cette combinatoire et c'est aussi chercher à en saisir la dynamique. En ce sens toute ville est nécessairement un objet unique. Dans cette perspective il est sans doute abusif de parler de ville du Sud comme d'ailleurs de ville du Nord. Cependant, au-delà de cette extraordinaire diversité urbaine il est sans doute possible de repérer certaines régularités ou à tout le moins les différents éléments de la combinatoire et les modalités spécifiques selon lesquelles ils s'articulent.

Dans la société issue de la société industrielle les différentes logiques qui travaillent la ville tendent à présenter certaines caractéristiques communes

La logique économique. Comme l'a très bien montré Henri Lefebvre la ville dans la société capitaliste se trouve affecté par les rapports socioéconomiques dominant. La valeur marchande tend à y prendre une place de plus en plus centrale. L'hégémonie de plus en plus forte de cette dimension marchande conduit à réduire l'espace ou d'autres dimensions pouvaient se déployer. Dans la ville antique et dans la ville médiévale la ville n'est que très peu un espace doté d'une valeur économique. Cette affirmation peut paraitre paradoxale dans la mesure où la ville fut par excellence un lieu de production et d'échanges économiques. Au cœur de la ville romaine, près du forum se tient le marché et c'est bien dans la ville médiévale que se concentrent les acteurs économiques, cette bourgeoisie qui constituera la force essentielle du développement économique. Mais si la ville 
est le lieu de l'économique elle n'est pas un objet économique, à proprement parler l'espace n'a pas de valeur marchande. Patriciens romains ou bourgeois médiévaux dépensent beaucoup pour embellir la ville. Certes cet investissement n'est pas gratuit, il constitue un moyen d'asseoir sa puissance, de la montrer, mais cette pratique ostentatoire passe par la réalisation de la ville comme œuvre. La production monumentale a quelque chose d'à la foi grandiose et gratuite. Et dans le Moyen Age occidental l'église va aussi jouer un grand rôle dans cette démarche ostentatoire. A travers la cathédrale il s'agit certes de rendre hommage à Dieu mais il s'agit aussi de montrer la puissance de l'Eglise et pour les nobles et les bourgeois qui financent de s'assurer une chance de Salut. En ce sens la dépense monumentale peut être considérée comme un investissement dont on attend un retour mais celui-ci n'est jamais principalement monétaire. Dans cette démarche le Beau tient une place tout à fait importante. Plus beau, plus grand tel est le critère qui marquera la grandeur de celui qui investit.

Avec l'avènement du capitaliste l'espace urbain va devenir objet de transaction, il va acquérir une valeur marchande. Aujourd'hui le foncier est l'objet de luttes spéculatives féroces, l'espace est un marché et produire la ville est de plus en plus une activité économique qui se distingue de moins en moins des autres activités économiques. Une grande partie de l'espace urbain bascule dans le champ du marché immobilier où circulent des capitaux considérables. Ce phénomène s'observe partout. Les crises récentes ont montré sa prégnance aux Etats-Unis, en Chine, en Espagne mais c'est un phénomène généralisé.

La réduction de l'espace urbain à sa dimension marchande a des conséquences très précises. La plus évidente c'est la réduction de la dimension proprement urbanistique dans la production de la ville. Là où l'investissement s'avère le plus rentable c'est la construction immobilière, d'où une dynamique de densification extrême des zones ou le cout du foncier est le plus élevé. La construction en hauteur s'impose, les zones non bâties tendent à se réduire au maximum. On assiste mécaniquement à une séparation fonctionnelle de l'espace extrêmement sévère partout où la logique économique parvient à dominer la configuration de production de l'espace urbain. Les quartiers où se multiplient les tours résidentielles à Belèm et dans tant de grandes villes brésilienne en sont une parfaite illustration.

Dans le même temps, les classes populaires sont reléguées dans des périphéries qui s'éloignent à mesure que la ville se développe. Subsistent alors de vastes zones où des logiques culturelles traduites dans l'espace urbain conditionnent la production de cette espace où l'auto construction est très présente (Agier 2009) Ce phénomène est tout à fait significatif dans les villes du 
Sud où les inégalités économiques présentent une amplitude particulièrement importante. Cependant, cette logique économique n'est jamais totalement libre de s'imposer. Elle se trouve bornée par la logique politique.

La logique politique. La ville de tout temps et ce depuis son apparition au néolithique est un lieu éminemment politique. Elle est même fondamentalement le lieu du politique. Avec la ville, en même temps que s'accentue considérablement la division du travail que naît l'artisanat le pouvoir politique apparait. Il est à la fois le garant de l'ordre intérieur, celui qui maintient la cohérence entre ces nouvelles composantes de la société, et celui qui, en tant que chef militaire, garantit la sécurité vis-à-vis de l'extérieur. La ville en effet dès le néolithique se protège derrière ses murs. C'est dans la ville que naît le politique proprement dit comme le souligne Gordon Childe en parlant de révolution urbaine. Le politique est donc d'abord un phénomène urbain, et c'est d'abord sur la ville qu'il va chercher à exercer son autorité, à manifester sa puissance. Ce phénomène évident dès les villes de la Mésopotamie ou de l'Egypte pharaonique atteindra une première apogée avec Rome. La puissance politique de Rome, la capacité du politique à s'imposer dans une configuration particulière de centralisation et de concentration des pouvoirs, rappelons que l'Empereur est Imperator donc chef militaire et aussi grand pontife donc maitre du religieux. Dans cette configuration pouvoir politique, militaire et religieux se trouvent concentrés en une seule personne ce qui donne cette extraordinaire puissance du politique à Rome. C'est cette puissance du politique qui lui donne la capacité de maitriser et d'organiser l'urbain. La ville contribuera à l'affirmation de cette puissance du politique. La ville romaine va s'installer partout sur le pourtour méditerranéen. Elle rend visible partout la domination de l'empire romain. Le politique s'incarne dans une symbolique urbaine. Amphithéâtres, thermes temples, forum sont autant de marqueur de la toute-puissance romaine. Mais le politique n’impose sa marque que dans le cadre d'un Etat fort, ou, à tout le moins d'une puissance politique forte. Cette force du politique pose les bases de la possibilité d'un encadrement normatif de la ville, principalement à partir du droit. L'urbain se présente toujours comme un espace investi paf le droit. Pas d'urbain sans règlementation. Mais la capacité de cette règlementation à s'incarner dans l'espace est très largement fonction du statut du politique dans la société. La place du politique dans la configuration des différentes logiques détermine sa capacité à s'imposer comme instance de normalisation des pratiques et des comportements.

Sur ce dernier point les différences entre les villes du Sud et les villes du Nord sont tout à fait considérables. Il ne faut en effet pas confondre puissance du politique et Etat autoritaire ce que l'on fait trop souvent. Beaucoup de pays du 
Sud, notamment en Afrique, mais pas seulement, se caractérisent en effet par des régimes autoritaires voire dictatoriaux dans lesquelles la démocratie quand même elle existe n'est que de façade mais cela ne signifie pas que le politique y soit puissant. La puissance du politique dépend en fait d'une dimension particulière où s'articulent les différentes logiques. C'est le rapport entre logique politique et logique traditionnelle qui établit la capacité du politique à imposer ses décisions à un ensemble social. Autrement dit un système peut avoir toutes les apparences d'un modèle rationnel-légal sans pour autant que ce modèle soit capable d'imposer sa logique à la société civile. Les pays du Sud, notamment en Afrique, sont à cet égard dans une situation paradoxale, l'Etat y est fort et la puissance publique faible. Ce sont des Etats autoritaires dans lesquels la démocratie est restreinte, mais où le système politico administratif est faible, incapable d'imposer ses règles sur l'ensemble du territoire, contraint de composer avec les autorités traditionnel. En cas de crise grave ce pouvoir comme le montre les crises actuelles en Afrique se délite très rapidement incapable d'imposer son autorité. Des pays émergents, comme l'Inde ou le brésil, qui sont des démocraties, se présentent aussi comme des Etats relativement faibles. En effet, un Etat fort, c'est un Etat où la puissance publique à travers le relais un appareil bureaucratique légitime, est capable de s'imposer à l'ensemble du territoire. En France, par exemple, la démocratie est réelle, l'Etat n'est pas autoritaire mais la puissance publique est forte, elle peut faire respecter partout l'autorité de l'Etat.

Dans un Etat démocratique la configuration logique social logique politique logique économique se caractérise par une forte pression de la logique sociale sur la logique politique. Les mouvements sociaux exercent une forte pression sur le politique pour réduire les inégalités dans la ville et faciliter l'accès pour tous aux différents espaces urbains. Cette pression du social sur le politique brise le face à face du politique et de l'économique. Le politique doit intégrer dans sa démarche la demande social, au moins partiellement, s'il veut perdurer, être réélu. Il doit s'opposer à la toute-puissance de la logique économique, la réguler, lui imposer des règles.

La situation est tout autre dans les Etats autoritaires du Sud où c'est exclusivement le monde économique qui fait pression sur la politique, notamment par le moyen de la corruption des élites politiques. Cela se vérifie partout et semble-t-il aussi au Brésil.

Enfin la ville est bien l'inscription dans l'espace des rapports sociaux dominants et particulièrement des rapports d'inégalité et des formes spécifiques que ceux-ci prennent selon les pays. Toutes les sociétés, au Nord comme au Sud sont des sociétés inégalitaires, cependant l'amplitude des inégalités varie beaucoup d'un pays à l'autre. Plus généralement cette amplitude est beaucoup 
plus importante au Sud qu'au Nord et c'est cette différence qui s'incarne dans l'espace. Cela produit une sorte de tripartition de l'espace urbain. Le sommet de la pyramide sociale s'enferme dans des quartiers protégées, ultra-sécurisés dans un phénomène de gentrification bien connu, le cœur de l'espace urbain lui est soumis à une forte domination des logiques économiques dans le cadre d'un modèle urbanistique internationale ce qui produit le phénomène d'uniformisation de l'urbain que l'on observe à Shanghai, à Dakar et dans de très nombreuses villes brésiliennes. Enfin les classes les moins favorisée se regroupent dans des quartiers qui échappent à ce schéma d'urbanisation. Le creusement des inégalités sociales, partagé par les sociétés du monde en développement, s'exprime avec une intensité particulière en milieu urbain. Dans des économies fragiles, face à la précarité grandissante de 1 'emploi, le logement apparaît comme un refuge pour les familles pauvres comme pour les classes moyennes paupérisées par des crises économiques. Dans un contexte de pauvreté de plus en plus présente en ville et d'insuffisance de logements sociaux, la question du logement est largement résolue par les habitants eux-mêmes, à travers l'auto-construction, le plus souvent sur des terrains occupés ou construits illégalement. Ces espaces répondent à d'autres logiques. On y observe notamment des mécanismes de reconstitution des logiques traditionnelles portées par les modèles culturelles des populations qui les occupent. Néanmoins ces logiques traditionnelles présentent des formes différentes de ce qu'elles sont dans l'espace rurale d'où elles sont originaires. Sous des formes spécifiques elles se combinent néanmoins aux logiques économiques et politiques qui traversent l'espace urbain.

Ces modes d'organisation correspondent à des moments différents du processus d'évolution urbaine. Il faut en effet souligner avec force combien la société urbaine engendré par la révolution industrielle est aussi une révolution culturelle. C'est à bon droit qu'Henri Lebvèvre, peut parler de Révolution Urbaine pour qualifier cette profonde transformation culturelle que porte la ville née de la révolution industrielle. L'expression permet de saisir qu'il s'agit en même temps que de profonde transformations dans la ville d'une véritable révolution culturelle. Certains traits de cette révolution ont été bien analysés par G.Simmel [Simmel 1998] dès la fin du $19^{\text {m̀me }}$ siècle et bien sûr en sociologie par l'Ecole de Chicago.

L'émergence de la société urbaine se traduit par de profondes transformations des modèles culturelles de références et des valeurs qui leurs sont associées. Passage progressif d'un modèle ou le collectif l'emporte sur l'individu à un modèle ou au contraire c'est l'individu qui l'emporte d'un modèle altruiste à un modèle égoïste pour parler comme Durkheim [Durkheim, 1897]. Dans les pays du Nord ce processus est enclenché depuis le début du $19^{\text {ème }}$ 
siècle et il a profondément bouleversé ces sociétés même si il peut cependant encore rencontrer des formes de résistances populaires, c'est ainsi qu'on assiste en France et en Europe depuis plus de vingt ans à des formes diverses de repli identitaires [Plas 2010] Dans les pays du Sud en général et spécifiquement en Afrique, le processus est moins avancé. Mais de ce point de vu le Sud tend de plus en plus à rompre son unité avec le développement du phénomène des pays émergents comme la Chine, l'Inde ou le Brésil dans lesquelles le processus au contraire s'accélère.

Le passage des sociétés à dominante rurale à des sociétés à dominante urbaine correspond à un phénomène de désagrégation culturelle et de reconstruction progressive d'un nouveau modèle culturelle. Ce processus s'effectue en articulant des éléments de la société urbaine qui présentent une relative universalité avec des traits propres aux différentes aires culturelles. Ce sont les modalités particulières de cette articulation ainsi que la diversité des cultures incorporées qui produisent les différentes formes d'organisation sociétales et les différentes formes d'organisation urbaine. Dans l'action locale on observe comment des acteurs traditionnels s'emparent de mécanismes institutionnels nouveaux pour négocier leur spécificité [Teisserenc 2013]

Cependant en règle générale, dans les pays du Sud émergents on observe une urbanisation rapide dominée par la logique économique. La conséquence en est un urbanisme vertical très dense dans lequel on assiste à une accélération du processus de désagrégation des modèles culturelles traditionnels ce qui se traduit notamment par un très important développement des violences urbaines. Cellesci sont la conséquence d'un double mécanisme d'une part de la progression des valeurs d'individualisme, des logiques de réussite individuelle et, d'autre part de l'affaiblissement du contrôle social qu'exerçaient les sociétés traditionnelles. Le moteur de ces violences est le même que dans la logique économique comme l'avait bien vu Merton [Merton 1953]. Les valeurs sont les mêmes : concurrence, lutte pour accéder aux biens reconnus mais les moyens sont considérés comme illégitimes.

\section{CONCLUSION}

La ville du sud dans les pays émergents est une ville en transition, elle cherche des modalités propres qui lui permettent de produire une modalité particulière de la société urbaine. Ville jeune elle manifeste un dynamisme très puissant ou se mêle souvent quête démocratique et volonté de perpétuer des formes traditionnelles de sociabilité. C'est aussi une ville en danger. L'enjeu est considérable de savoir dans quelle mesure les logiques sociales et politiques parviendront à rééquilibrer la logique économique aujourd'hui dominante. 


\section{REFERENCES}

AGIER, M. Esquisses d'une anthropdogie dela ville. Lieux, sifrations, mouvements. Louvain-la-neuve, Academia - Bruylant.

CHILDE, G. (1925). La naissance de la civilisation européenne. [s.l]: Kontre Kulture, 2013.

DUBY, G. Histoire de la France urbaine. Paris: Seuil, 1980.

DURKHEIM, É. Le Suicide: étude de sociologie. Introduction de Serge Paugam.

GUILHOT, N. Sciences Humaines et Sociales. Paris: La Découverte, 2005.

LATOUR, B. Changer de société, refaire de la sociologie. Paris: La Découverte, 2006.

LE GOFF, J. (1898). La ville en France au Moyen Age. Des Carolingiens à la

LEBVÈVRE, H. La Révolution urbaine. Paris: Gallimard, 1970. (Collection Idées).

LEBVÈVRE, H. (1974). La production de l'espace. Paris: Anthropos, 2000. 485 p. (Coll. Ethnosociologie).

LEBVÈVRE, H. Le Droit à la ville. 2. ed. Paris: Anthropos; Seuil, 1968. (Collection Points)

MERTON, R. K. (1953). Éléments de théorie et de méthode sociologique. 2. ed. Plon: [s.n.], 1965.

Paris: Presses Universitaires de France, 2007. (Coll. Quadrige/Grands Textes)

PLAS, P. Le double repli identitaire des classes populaires, Esprit critique été, v.13, n.113, 2010.

Renaissance. Paris: Seuil, 1998.

SANTOS, M. Dix essais sur les villes des pays sous-développés. Paris: Éditions Orphys, 1970. $121 \mathrm{p}$.

SANTOS, M. Les villes du Tiers monde. Paris: Génin Librairies Techniques, 1971. $428 \mathrm{p}$.

SIMMEL, G. Philosophie de la modernité 1: la femme, la ville, l'individualisme. Payot: [s.n.], 1988.

SYLVAIN, A. Les villes en mutation. L'Économie Politique, v. 1, n. 53, 2012.

VELTZ, P. Mondialisation, villes et territoires: une économie d'archipel. Paris: PUF, 2014. (Coll. Quadrige).

VÉRON, J. L'urbanisation du monde. Paris: La Découverte, 2006. 122 p. (Coll. Repères). 


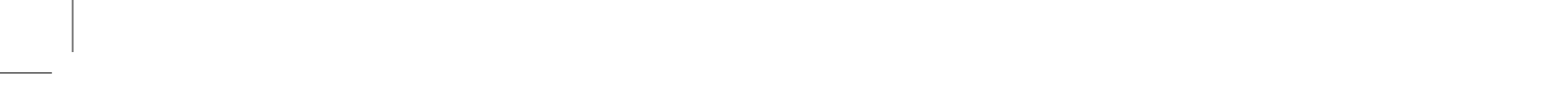

